\title{
ANTI-MYCOBACTERIAL ACTIVITY OF CAPPARIS SPINOSA L. EXTRACT AGAINST CLINICAL ISOLATES OF MYCOBACTERIUM TUBERCULOSIS
}

\author{
AZADEH EHSANIFAR ${ }^{1}$, NADIA KAZEMIPOOR ${ }^{1}$, BAHMAN FOULADI ${ }^{2 *}$ \\ ${ }^{1}$ Department of Microbiology, Islamic Azad University of Kerman Branch, Kerman, Iran. ${ }^{2}$ Department of Mycology and Parasitology, Zabol \\ University of Medical Sciences, Zabol, Iran. Email: fouladibahman@yahoo.com
}

Received: 09 February 2017, Revised and Accepted: 24 April 2017

\section{ABSTRACT}

Objective: Tuberculosis (TB) has been one of the biggest global health issues for decades. Emerging forms of the disease; however, after many years since the discovery of the last effective treatment for this disease, the emerging forms of it have made scientists worried about the disease. Most herbs have anti-microbial properties; however, there was no research on the impact of fruit Capparis spinosa on TB. This study was conducted to evaluate the anti-microbial effects of bacterial C. spinosa.

Methods: In this study, methanolic extract at maximum efficiency to maintain existing substances was prepared by the freeze dryer device and to determine the minimum inhibitory concentration of bacteria's growth. The proportional method was used on 7 clinical samples and one reference sample H37RV with its six concentrations (80-50-25-10-1-0/2 mg/ml). In addition, to determine the effects of plant extracts in combination with rifampin, all samples were tested by the supplement combination or cover combination of these two materials. All tests were performed twice. The methanol extract of the plant at the concentration of $25 \mathrm{mg} / \mathrm{mL}$ in all samples has inhibitory effect on the growth.

Results: In study, clinical samples with a lethal dose of RIFAMPIN, it was shown that none of the samples have drug resistance. The results have revealed that the methanol extract has an anti-mycobacterial effect.

Conclusion: The results of this study indicate that Capparis spinosa possess considerable anti-mycobacterial properties in the laboratory. These results can be used as a background for further future studies on this species.

Keywords: Mycobacterium anti-bacterial, Plant Capparis spinosa, Minimum inhibitory concentration

(c) 2017 The Authors. Published by Innovare Academic Sciences Pvt Ltd. This is an open access article under the CC BY license (http://creativecommons. org/licenses/by/4. 0/) DOI: http://dx.doi.org/10.22159/ajpcr.2017.v10i8.17629

\section{INTRODUCTION}

Tuberculosis (TB) is a social disease with the medical features which is described as a barometer of social welfare [1] TB, the biggest factor causing death among infectious disease in the world (even more than AIDS, malaria and measles), ranks tenth in the global burden of disease 2020 and is expected to maintain its current position or rise to the seventh position [2].

In Africa, many plants are used in traditional medicine to treat TB and other respiratory diseases. The emergence of drug resistance and its conversion into a major threat require an urgent search for anti-TB agents which are effective and safe to consume [3].

TB is a disease caused by a known factor and known epidemiology and its treatment principles are known for about 60 years, and also it is more than a quarter of a century that a short-term treatment regime is used for it; however, some patients are still undiagnosed or not treated appropriately. Unfortunately, the results of these shortcomings and at the same time the increasing incidence of HIV, prepare the conditions for emergence, and spread of multidrug-resistant TB bacillus [2].

Mycobacterium is an aerobics rod-shaped bacillus which does not form spore and gain its energy from simple carbon compounds' oxidation. Moreover, the biochemical activity of bacteria is very slow. They have high chemical agents' resistance than other bacteria, due to the hydrophobic nature of the mycobacteria surface and their mass growth [4]. Drug-resistant strains pose a serious challenge to global health, in addition to their need for a long-term treatment; they also have side effects [5]. Therefore, the use of herbal remedies as an alternative or complementary for industrial medications gainsa considerable importance [6]
Capparis spinosa L. (Capparaceae; common name: Caper) is a plant from the 1-year herbaceous plant families that grows as shrubs and often covered with glandular. Studies conducted in most parts of the world have been found that most species have anti-bacterial effects and natives consume some species as a traditional medicine. This plant grows in the most parts of the Middle East a wild plant, but not much research has been done on it [7].

Herbal medicines have been used as a non-comprehensive metabolite for pharmaceutical development also as an alternative treatment for human diseases [5]. They contain many active components that have therapeutic value [8]. Iran is a vast country and as a result of its geographical situation on earth, it is a confluence point of several different climates; thus, owns various weathers. These climatic conditions can cause rare plant flora on this country. Phytochemical studies on 1700 different plants show that Iran's plants possess very good quality and quantity [9].

This Capparaceae have 30 genera and 650 species, and they grow mainly in warm and temperate areas and grow like shrubs [10]. Their bushes are crawling with long life and downy, and they have ramified branches with 1-1.5 m length. This plant has a long root that penetrates soil deeply. Its large flowers have white. Fruits come in different ways such as capsule, pod, and broken shafts and seeds are without albumen content. It has a long excrescence (Receptacle), at the end of itself which is light green at the beginning but gradually turns into red. Flowering time continues from spring to the late summer. In different plantation areas, this plant has a wide distribution. It grows well in sabulous lands. All parts of the plant such as fruits, roots, bark, leaves, buds, and stems can be used.

Its fruit graded by the size in 8 different sizes and export from Iran with the best quality. This plant tolerates $40^{\circ} \mathrm{C}$ on summer, and due to its 
tendency toward the cold weather, it can tolerate $-8^{\circ} \mathrm{C}$ on winters. It more likes the calcareous soils. Although this plant is cold in nature, it grows even in the dry and nutrient poor soils. Flowers grow on the $1^{\text {st }}$-year branches and they opened by the sunrise and closed by sunset. Its flowers should be harvesting before unfolding. At the end of season plant's fruit will split and seeds fall. Capparis has several chemical active compounds but the important one is flavonoid. Roots and flower buds contain pectin, saponin, aresin material, aminoglycosides, and substance called kaparyroutin. Octadecadienoic acid-hexadecanoic acid-octadec9-enoic acid- 1, 2 - benzene dicarboxylic acid-bis(2-methylpropyl) ester- (2ethylhexyl) phthalate-2-methoxy-phenol- tetradecanoic acid - dodecanoic acid -z,z- 9 - 12 - octadecadienoic acid-ethyl ester -1-(1H-pyrrol-2-yl) - ethanone - hexadecanoic acid -methyl ester [11].

The first use of this herb for medicinal purposes assigned to the Sumerians. In the traditional medicine, it is used as support and maintenance, liver stimulant, and it improves the function of the liver. In addition, it is used to reduce bloating, atherosclerosis of the vessels, and as an anti-rheumatic. Moreover, it is reported as a worm excretion factor, diuretic, and nutrition [12].

In traditional medicine, the decoction of the root's bark is utilized to heal the accumulation of serous fluid in the cavities and cure anemia, arthritis, and gout. In addition, in previous studies, anti-TB property of this pant has been reported. In addition, recent studies confirm its beneficial effect on hysteria and neural states [12].

\section{METHODS}

Referring to the suburbs and surrounding villages in Sistan, wild Capparis spinosa samples were collected in July 2015. The fruits were then washed and dried in the shade.

\section{Preparation Capparis spinosa fruit extracts}

Of the $300 \mathrm{~g}$ from powder measured by the ratio of 1-5 was dissolved in methanol. It was then placed on a timer shaker for $48 \mathrm{hrs}$ at $150 \mathrm{rpm}$, then filtered out by filter paper and poured over the three petri dishes. To accelerate the sublimation of methanol, the samples were incubated at $40^{\circ} \mathrm{C}$. After 3 days, the samples were placed in a freeze dryer.

\section{Microorganism preparation}

The sample were collected from Sistan and Baluchestan urban and rural health-care centers and sent to test for TB to the Southeast of the country. The obtained samples from medium cultured on Lowenstein-Jensen culture Media. After 6 weeks, the pipes were assessed and it was clear to see that the mycobacterium colonies and the medium were half-covered. A Ziehl-Neelsen test was performed for recognition.

For reference, culture H37RV standard samples were used. Standard samples were collected from the Tehran reference TB. All seven clinical samples were tested to determine the species. First, we had to determine the growth rate. For this purpose, prepared culture medium on Lowenstein-Jensen culture medium and it was evaluated daily for the presence of colonies. The growing temperature was assessed, and for this purpose 5 different places were prepared and incubated at 44-42-37-33-30-25 temperature. Samples were examined for colonies on days 3-7-14-21. The colony morphology was assessed and a pigment production test was performed. To do this, both samples were inoculated in 2 different mediums. One was then covered with aluminum foil and incubated for 18 days at $37^{\circ} \mathrm{C}$. Next, a nitrate resuscitation test was conducted and the number of colonies of each sample added separately to $0.1 \%$ sodium nitrate solution and incubated for $4 \mathrm{hrs}$ in Ben Murray at $37^{\circ} \mathrm{C}$ then 2 drops of normal hydrochloric acid, 2 drops of sulfanilic acid $0.2 \%$ and 2 drops the solution $\mathrm{N}$-1-naphthyl diethyl diamine hydrochloride $0.1 \%$ added to tube and color examined. Next stage testing niacin, for this test about the $1 \mathrm{ml}$ of Sterile Distilled water poured into medium with 50 colonies at least, waiting for 30 minutes and then, $0.5 \mathrm{~mL}$ of solution of top on the medium transferred to sterile tube. $0.5 \mathrm{~mL}$ of Aniline represented $4 \%$ and $0.5 \mathrm{~mL}$ of cyanogen bromide reagent added to tube, color examines helped diagnosis.

\section{Preparing Levin-Stein Johnson vitro containing Capparis spinosa fruit extract}

All the test equipment was sterilized using autoclave at a temperature of $121^{\circ} \mathrm{C}$ at 15 minutes and cooled by keeping them at room temperature. To make the egg suspension, first the eggs were cleaned with a brush and placed in a soap solution for 1-5 minutes. Then, these were washed in cold water and placed in $70 \%$ alcohol for 15 minutes. After being washed by hand in soap and water, the eggs were broken into a sterile electric mixer container and blended smoothly. The resulting mixture was passed through four layers of typical sterile filter cloth and poured into a $2 \mathrm{l}$ jar. A salt solution was made next by adding malachite green $2 \%$ solution. After adding the salt solution and malachite solution to the eggs, they were placed on a hot plate so they could become mixed [10].

To prepare the fruit juice, a concentration of $C$. spinosa and inoculated that to the under making medium. The weight of the empty plate was calculated. Then, after adding some extract and reassessing the weight of the plate, $30 \mathrm{mg}$ dimethyl sulphoxide was added to dissolve completely on $25 \mathrm{mg}$ of extract solvent. The egg solution was divided into $150 \mathrm{~mL}$ in 6 different containers. Using $\mathrm{C}_{1} \mathrm{~V}_{1}=\mathrm{C}_{2} \mathrm{~V}_{2}$ formula and adding the extract to a Lowenstein-Jensen solution, $150 \mathrm{mg} / \mathrm{mL}$ concentration was gained. Then, 8-6 $\mathrm{mL}$ of each concentration was poured into a McCartney tube and the lid tightly closed. This was flocculated diagonally on a coagulator at $85^{\circ} \mathrm{C}$ for 50 minutes and then, placed in an incubator at $37^{\circ} \mathrm{C}$ for $24 \mathrm{hrs}$. After health checking, it was placed in a refrigerator with a temperature of $2-8^{\circ} \mathrm{C}$.

\section{Prepare the witness vitro and positive control to determine the} possible resistance of microbes

A medium was prepared without any additive for witness purposes. In addition, to ensure that the samples were not resistive, all clinical samples, and reference samples had two witness tubes without the extract and contained anti-mycobacterium. On day two, the mediums were examined for steam and contamination and then, carried to the microbial culture room [3]

\section{Microbial stoke and culture preparation}

First, the cabinet was disinfected with $25 \mathrm{~mL}$ formaldehyde and $15 \mathrm{~g}$ potassium permanganate. Then, the hood was turned on and the culture began 30 minutes later. Eight McCartney tubes with 8-10 sterile Pearl Glassatroom temperature was taken and with disposable sterile lobe, one complete lobe (8-10 colonies) were moved from the medium of the first colonies to the tube and the lid was closed tightly and placed on the shaker for 4-5 minutes. Then, after waiting for 5 minutes, the tube lid was opened. 2cc distilled water was added and the lid was closed again and shaken for 4-5 minutes, then rested for 45 minutes so that the tube contents remain stagnant. The procedure was repeated for each sample and reference sample.

During the next stage, we created a McFarlane. Sulfuric acid 1\% $(990 \mathrm{~mL})+1 \%$ barium chloride $(10 \mathrm{~mL})$. Then, with sampler head lifting $0.1 \mathrm{~mL}$ of top of liquid, the colony suspension was added to the tube with $1 \mathrm{cc}$ distilled water; by adding distilled water it matched a McFarlane. $500 \mu \mathrm{l}$ of suspension were picked up with the sampler and added to the $4.5 \mathrm{~mL}$ distilled water tube and diluted 10 to the power of minus one. Then, $0.1 \mathrm{~mL}$ of the previous solution was added to $9.9 \mathrm{~mL}$ distilled water that created 10 to the power of minus two equivalent ( 3 multiplied by 10 to the power of 6 ) of bacteria on each $\mathrm{mL}$. Then, 1cc of stock solution was added to the prepared medium, and the cultured tubes were placed in an incubator at $37^{\circ} \mathrm{C}$ for 4 weeks.

The method used to determine the extract effect on the examined samples was the proportional method. This method is a standard 


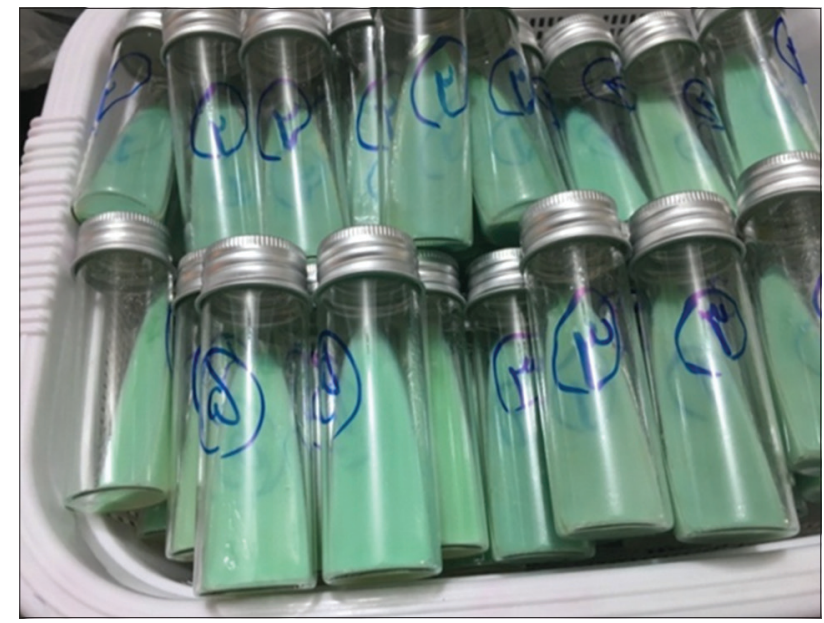

Fig. 1: Lowenstein-Jensen medium containing Capparis spinosa methanolic extract

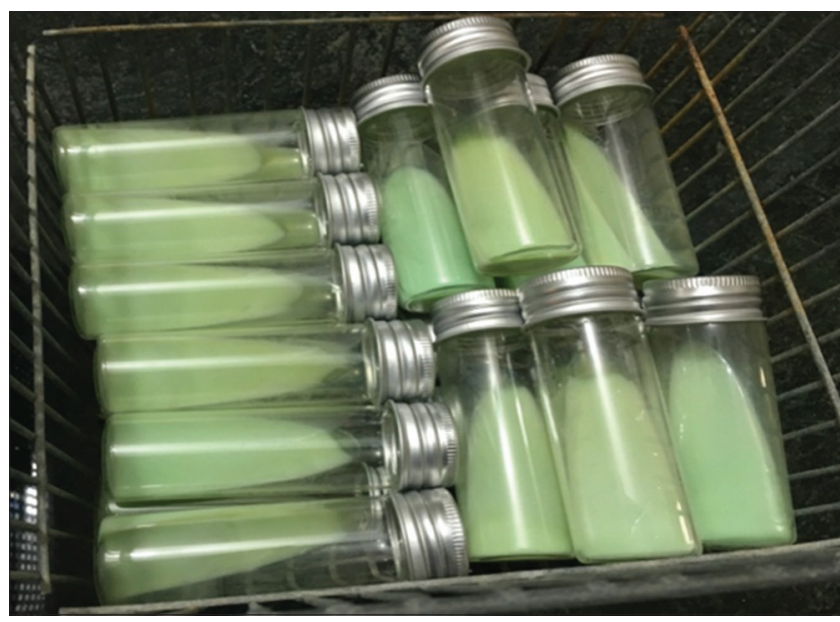

Fig. 2: Normal Lowenstein-Jensen medium

procedure and is approved by the World Health Organization and Clinical and Laboratory Standards Institute [13]. This method needs 3-4 weeks' incubation.

\section{RESULTS}

Based on species diagnostic tests at the end all of samples were reported as Mycobacterium TB. After adding brown extract to Lowenstein-Jensen medium, medium color changed from green inclined white to dark green.

The statistical examination of this study was carried out using the normality check of the Kolmogorov-Smirnovtest, while the Significance level was checked by the Spearman Kendall test.

\section{DISCUSSION}

During this research, the methanol extract of $C$. spinosa was prepared using the maceration method and freeze dried. 7 clinical samples and one Mycobacterium TB sample were isolated and identified. According to the results, the $C$. spinosa extract with $25 \mathrm{mg}$ on $\mathrm{mL}$ minimum inhibitory concentration (MIC) was effective on 6 clinical samples. On the second sample, with $10 \mathrm{mg}$ on $\mathrm{mL}$ MIC of methanol, C. spinosa fruit extract was effective. The H37RV standard strain culture result with $25 \mathrm{mg}$ on mL MIC of methanol C. spinosa fruit extract was effective. It is most probable that the freeze-drying method has the best stability on the known and unknown material of $C$. spinosa fruit extract, according the extract methods and their impact on the stability of the effective plant compounds.

This research used the best method to preserve plant properties. According to research conducted Institute of Medicinal Plants (SID), compared with other methods of extraction, such as thermaldryer, spray dryer, and rotary evaporator, using the freeze-dryer method has the most stability of medicine plants' known contents [14]. The other research is considered one of the first studies in Iran on medicine plants used on mycobacterium, who study shallot extract effects considered one of the first studies in Iran on medicine plants used on Mycobacterium. The results were effective on $0.1 \mathrm{mg}$ on $\mathrm{mL}$. Another study assessed the effect of ethanol on the growth of Mycobacterium TB strains. Based on this study, concentrations higher than $5.2 \%$ of ethanol prevented growth, but on $0.1-0.5 \%$ density has shortened growth time to 3-8 days. Assessing the antimicrobial effects of Salvadora persica water extract at Tehran's Medical University by Fallah et al. with $10 \mathrm{mg}$ on $\mathrm{mL}$ was effective [6].

In another study at the University of Spain in 2011, Lea et al. assessed Mozambique's traditional medicine for phytochemical properties. 15 medicinal plants extracts with hexane-dichloromethane-ethyl acetate - ethanol dissolver, at the same time a decoction of these plants was prepared for traditional use. In the face of two species, Mycobacterium asmgmatys H37RV with 1 inhibitory concentration $125 \mathrm{mg}$ on $\mathrm{mL}$ was effective [9]. Evaluated the toxicity and antiinflammatory genes of 10 plants used to cure TB in South Africa. Extraction was performed by 4 dissolvers, petroleum-etherdichloromethane-ethanol $80 \%$ water and using reference samples H37Ra. 6 plants with $1 \mathrm{mg}$ on $\mathrm{mL}$ density have suitable activity and water extract known worthless. An assessment of herbal medicines used for curing bacterial infectious diseases in Soudan was carried out by [15]. They assessed 50 extracts from 46 medicinal plants, extracted with hexane-chloroform-ethanol-ethyl acetate-n-butane and water. The results show that except for the water extracts, the rest of the extracts with $6.25 \mathrm{mg}$ on $\mathrm{mL}$ were effective. A study conducted by [16] in Ghana assessed the treatment of TB with 15 species of plants from 13 different genera. Among these plants, the Amaryllidaceae had the greatest anti-mycobacterium effect. A study in Canada analyzed the anti-mycobacterium effects of Aralia nudicaulis. The plant's methanol extract and H37Rv sample in liquid medium proved to be effective [17].

A survey conducted on the Citrullus colocynthis oil and chloroform and methanol extracts over the Mycobacterium other than TB sensitive and resistive strains, with 16 clinical samples in India by [17], was effective on $62.5 \mathrm{mg}$ on $\mathrm{mL}$ over sensitive strains and $125 \mathrm{mg}$ on $\mathrm{mL}$ for resistive strains.

In a study assessed the effect of additives monnocarbonyl analog of curcumin to prevent the growth of sensitive and resistant anti-TB drug strains. At Atlanta University, Baldwin et al. (2015) [18] carried out a disk diffusion method and liquid measuring on laboratory samples and resistant strain to rifampin. The synergistic effect was confirmed with rifampin. Because of poor bioavailability, it was not appropriate to use curcumin. By Singh et al. (2013) [19] screened for anti-mycobacterial activity of 5 herbal plants using a tube dilution method on M. asmgmatys and clinical strains. Prepared Hexan and methanol extracts were effective on 125 and $250 \mathrm{mg} / \mathrm{mL}$.

An overview study was conducted by Gautam et al. (2007) [7] on herbal medicines in India and their anti-mycobacterium effects. By diluted test method macro and micro agar for H37RV sample on middle brook medium and Alamar blue colorimetric system was used and received a wide result. This article reviews the research of 365 plant species between almost 17,500 plant species that show a wide range of antiMycobacterium TB. The most interesting part of this research correct confirmation of indigenous medicine and (yvrvdayy) about effective 
Table 1: The result MIC of methanol extract of fruits Capparis spinosa on 7 clinical sample and a standard sample and H37RV

\begin{tabular}{|c|c|c|c|c|c|c|c|c|c|c|c|c|}
\hline \multirow{2}{*}{$\begin{array}{l}\text { Extract mg on medium mL } \\
\text { First sample }\end{array}$} & \multicolumn{2}{|c|}{$80 \mathrm{mg}$} & \multicolumn{2}{|c|}{$50 \mathrm{mg}$} & \multicolumn{2}{|c|}{$25 \mathrm{mg}$} & \multicolumn{2}{|c|}{$10 \mathrm{mg}$} & \multicolumn{2}{|l|}{$1 \mathrm{mg}$} & \multicolumn{2}{|c|}{$0.2 \mathrm{mg}$} \\
\hline & 0 & 0 & 0 & 0 & 0 & 0 & 34.4 & 34.4 & 36.2 & 50 & 86.2 & 86.2 \\
\hline Second sample & 0 & 0 & 0 & 0 & 0 & 0 & 0 & 0 & 22.5 & 25 & 50 & 50 \\
\hline Third sample & 0 & 0 & 0 & 0 & 0 & 0 & 48.27 & 51.72 & 51.72 & 55.17 & 82.75 & 51.72 \\
\hline Forth sample & 0 & 0 & 0 & 0 & 0 & 0 & 40 & 50 & 40 & 40 & 80 & 92 \\
\hline Fifth sample & 0 & 0 & 0 & 0 & 0 & 0 & 17.14 & 18.57 & 57.14 & 57.14 & 71.42 & 85.71 \\
\hline Sixth sample & 0 & 0 & 0 & 0 & 0 & 0 & 6.66 & 10 & 83.33 & 91.66 & 83.33 & 100 \\
\hline Seventh sample & 0 & 0 & 0 & 0 & 0 & 0 & 33.33 & 33.33 & 41.66 & 46.66 & 83.33 & 100 \\
\hline
\end{tabular}

MIC: Minimum inhibitory concentration

Table 2: The relation between MIC significantly different from the concentration of the methanol extract of fruits Capparis spinosa marber clinical sample and a standard sample H37RV based on 7 compared to control medium

\begin{tabular}{lll}
\hline Statistical analysis & Group & Control \\
\hline Kendall's tau-b & & \\
$\quad$ Group & 1.000 & $-0.783^{* *}$ \\
$\quad$ Correlation coefficient & - & 0.000 \\
$\quad$ Significant (two-tailed) & 56 & 56 \\
$\quad$ N & & \\
Control & $-0.783^{* *}$ & 1.000 \\
$\quad$ Correlation coefficient & 0.000 & - \\
$\quad$ Significant (two-tailed) & 56 & 56 \\
$\quad$ N & & \\
Spearman's rho & 1.000 & $-0.906^{* *}$ \\
Group & - & 0.000 \\
$\quad$ Correlation coefficient & 56 & 56 \\
$\quad$ Significant (two-tailed) & & \\
$\quad$ N & $-0.906^{* *}$ & 1.000 \\
Control & 0.000 & - \\
$\quad$ Correlation coefficient & 56 & 56 \\
$\quad$ Significant (two-tailed) & & \\
$\quad$ N & & \\
\hline
\end{tabular}

MIC: Minimum inhibitory concentration, $* *<0.001$

or not been effective if this plants. A study in Brazil also assesses Mycobacterium TB. After obtaining novel derivatives of diospyrifoliumn plant leaves on 125-250 mg range was effective [20]. It also showed a synergistic effect with anti-TB drugs. In another study in South Africa by Dzoyem et al. [3], the anti-mycobacterium effect of 14 medicine plants was used on $2.6 \mathrm{mg}$ on $\mathrm{mL}$ density effected on M. smegmatis and Mycobacterium fortuitum.

A survey was conducted at the University of Cameroon by Nkenfou et al. [8] using the micro plate Alamar blue method. Experiments were carried out on 6 herbal plants on H37Rv reference sample with 31-125 $\mu \mathrm{g}$ on $\mathrm{mL}$. More over study by Jyoti et al. [21-23] in South Korea assessed the methanol extract of the Capillaris plant that contains ursolic acid on Mycobacterium TB. Clinical samples were placed on liquid medium and colorimetric and viewed under an electron microscope, with 4.1 and $5.1 \mathrm{mg}$ on $\mathrm{mL}$, density was effective.

The results of this study indicate that $C$. spinosa possess considerable anti-mycobacterial properties in the laboratory. These results can be used as a background for further future studies on this specie [20].

\section{REFERENCES}

1. Park K, Park J. Text Book of Preventive and Social Medicine Treatise of Community Health-Part II. Translation Shojaeetehrani H, Malekafzali H. Tehran: Nashre Samat; 2002.

2. Flores L, César P. Susceptibilidad antimicrobiana y patrones de resistencia de cepas sensibles de Mycobacterium tuberculosis en el establecimiento penitenciario lurigancho. Diagnóstico (Perú) 2011;50(2):77-83

3. Dzoyem JP, Aro AO, McGaw LJ, Eloff JN. Antimycobacterial activity against different pathogens and selectivity index of fourteen medicinal plants used in Southern Africa to treat tuberculosis and respiratory ailments. S Afr J Bot 2016;102:70-4.

4. Jawetz M, editor. Adelberg's Medical Microbiology. $24^{\text {th }}$ ed. Columbus, $\mathrm{OH}$ : McGraw-Hill Medical; 2007.

5. Sharifi-Rad J, Sharifi-Rad M, Hoseini-Alfatemi SM, Iriti M, Sharifi-Rad M, Sharifi-Rad M, et al. Composition, cytotoxic and antimicrobial activities of Satureja intermedia C.A. Mey essential oil. Int J Mol Sci 2015;16(8):17812-25.

6. Fallah M, Fallah F, Kamalinejad M, Malekan MA, Akhlaghi Z, Esmaeili M. The antimicrobial effect of aquatic extract of Salvadora persica on Mycobacterium bovis in vitro. Int $\mathrm{J}$ Mycobacteriol 2015;4:167-8.

7. Gautam R, Saklani A, Jachak SM. Indian medicinal plants as a source of antimycobacterial agents. J Ethnopharmacol 2007;110(2):200-34

8. Nkenfou CN, Mawabo IK, Notedji A, Nkenfou J, Fokou PV, Jouda JB, et al. In vitro antimycobacterial activity of six Cameroonian medicinal plants using microplate alamarBlue assay. Int $\mathrm{J}$ Mycobacteriol 2015;4(4):306-11.

9. Madikizela B, Ndhlala AR, Finnie JF, Van Staden J. Antimycobacterial, anti-inflammatory and genotoxicity evaluation of plants used for the treatment of tuberculosis and related symptoms in South Africa. J Ethnopharmacol 2014;153(2):386-91

10. Carpenter CD, O'Neill T, Picot N, Johnson JA, Robichaud GA, Webster D, et al. Anti-mycobacterial natural products from the Canadian medicinal plant Juniperus communis. J Ethnopharmacol 2012;143(2):695-700.

11. Heidari M, Bidoki K, Vafazade J. Evaluation of the Analgesic Effect of Methanolic Extract of Capparis spinosa in Mice. Persian [Dissertation] Kerman: Kerman Medical University; 2003. p. 44-7.

12. Rhizopoulou S, Ioannidi E, Alexandredes N, Argiropoulos A. A study on functional and structural traits of the nocturnal flowers of Capparis spinosa L. J Arid Environ 2006;66(4):635-47.

13. Nasiri MJ, Rezaei F, Zamani S, Darban-Sarokhalil D, Fooladi AA, Shojaei $\mathrm{H}$, et al. Drug resistance pattern of Mycobacterium tuberculosis isolates from patients of five provinces of Iran. Asian Pac J Trop Med 2014;7(3):193-6.

14. Ho MH, Kuo PY, Hsieh HJ, Hsien TY, Hou LT, Lai JY, et al. Preparation of porous scaffolds by using freeze-extraction and freeze-gelation methods. Biomaterials 2004;25(1):129-38.

15. Abuzeid N, Kalsum S, Koshy RJ, Larsson M, Glader M, Andersson H, et al. Antimycobacterial activity of selected medicinal plants traditionally used in Sudan to treat infectious diseases. J Ethnopharmacol 2014; $157: 134-9$

16. Nguta JM, Appiah-Opong R, Nyarko AK, Yeboah-Manu D, Addo PG. Medicinal plants used to treat $\mathrm{TB}$ in Ghana. Int $\mathrm{J}$ Mycobacteriol 2015;4(2):116-23.

17. Mehta A, Srivastva G, Kachhwaha S, Sharma M, Kothari SL. Antimycobacterial activity of Citrullus colocynthis (L.) Schrad. against drug sensitive and drug resistant Mycobacterium tuberculosis and MOTT clinical isolates. J Ethnopharmacol 2013;149(1):195-200.

18. Baldwin PR, Reeves AZ, Powell KR, Napier RJ, Swimm AI, Sun A, et al. Monocarbonyl analogs of curcumin inhibit growth of antibiotic sensitive and resistant strains of Mycobacterium tuberculosis. Eur J Med Chem 2015;92:693-9.

19. Singh R, Hussain S, Verma R, Sharma P. Anti-mycobacterial screening of five Indian medicinal plants and partial purification of active extracts of Cassia sophera and Urtica dioica. Asian Pac J Trop Med 2013;6(5):366-71

20. Regiane BD, Espelho SC, Pires CT, Garcia VA, Cardozo-Filho L, Cortez LE, et al. A new benzoic acid derivative from Piper diospyrifolium and its anti - Mycobacterium tuberculosis activity. 
Phytochem Lett 2015;11:18-23.

21. Jyoti MA, Nam KW, Jang WS, Kim YH, Kim SK, Lee BE, et al. Antimycobacterial activity of methanolic plant extract of Artemisia capillaris containing ursolic acid and hydroquinone against Mycobacterium tuberculosis. J Infect Chemother 2016;22(4):200-8.

22. Vijayasri S, Archana H, Agrawal A, Hopper W. Computational analysis of interactions between anti-epileptic drugs and important placental proteins-a possible route for neural tube defects in humans. Int J Pharm Pharm Sci 2016;8:19-23.

23. Kaur A, Thatai P, Sharma S, Sapra B. Green tea extract in microemulsion: Stability, dermal sensitization and efficacy against uv induced damages. Int J Pharm Pharm Sci 2016;8:1-8 